\title{
HMI Development for a Purpose-Built Electric Taxi in Singapore
}

$\begin{array}{ll}\begin{array}{l}\text { Sebastian Osswald } \\ \text { TUM CREATE } \\ 1 \text { Create Way, Singapore }\end{array} & \begin{array}{l}\text { Martin Schaller } \\ \text { TUM CREATE } \\ 1 \text { Create Way, Singapore }\end{array} \\ \begin{array}{l}\text { Daniel Zehe } \\ \text { TUM CREATE } \\ 1 \text { Create Way, Singapore }\end{array} & \begin{array}{l}\text { Stephan Schickram } \\ \text { TUM CREATE } \\ 1 \text { Create Way, Singapore }\end{array} \\ \begin{array}{l}\text { Philipp Mundhenk } \\ \text { TUM CREATE } \\ 1 \text { Create Way, Singapore }\end{array} & \begin{array}{l}\text { Daniel Gleyzes } \\ \text { TUM CREATE }\end{array} \\ \text { Pratik Sheth } & \text { Firstname.Lastname@tum- } \\ \text { TUM CREATE } & \text { create.edu.sg } \\ 1 \text { Create Way, Singapore } & \end{array}$

Permission to make digital or hard copies of part or all of this work for personal or classroom use is granted without fee provided that copies are not made or distributed for profit or commercial advantage and that copies bear this notice and the full citation on the first page. Copyrights for third-party components of this work must be honored. For all other uses, contact the Owner/Author.

Copyright is held by the owner/author(s).

\begin{abstract}
In this paper, we describe the development process of a human-machine interface (HMI) for a purpose-built electric taxi in Singapore. Based on the requirements of taxi drivers and passengers, we developed an automotive communication structure to support the connectivity between the vehicle HMI and mobile devices, support taxi-related functionalities and emphasize the characteristics of the electric vehicle. We carried out seven requirement studies in Singapore, implemented an integrated HMI platform, and developed different interface prototypes for the vehicle HMI and smart devices. This work contributes to the emerging field of automotive user interfaces by proposing a fully integrated HMI for an electric taxi.
\end{abstract}

\section{Author Keywords}

Electric vehicle; taxi; HMI development; requirement engineering; human factors; development platform.

\section{ACM Classification Keywords}

H.5.2. Information interfaces and presentation (e.g., $\mathrm{HCI}$ ): User Interfaces.

\section{General Terms}

Human Factors, Design.

MobileHCI '13, Aug 27-30 2013, Munich, Germany ACM 978-1-4503-2273-7/13/08.

http://dx.doi.org/10.1145/2493190.2494089 


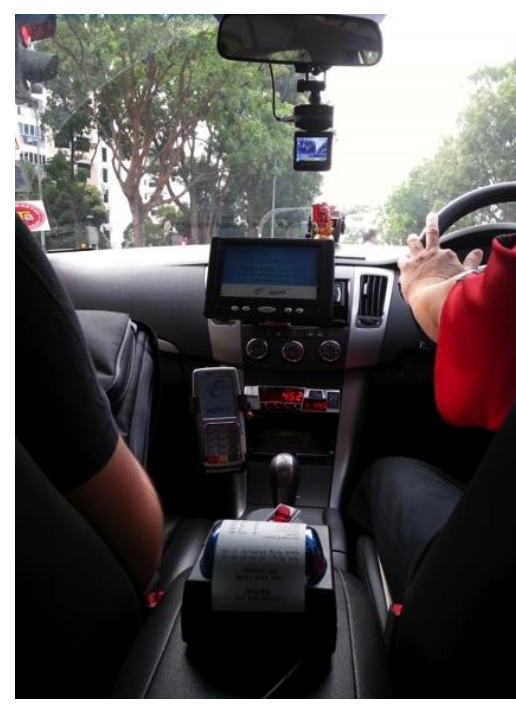

Figure 1: Interior of a taxi in Singapore

\section{Introduction}

Advanced automotive human-machine interfaces (HMI) are becoming widely available in current vehicles [2][6]. The information provided ranges from entertainment and driver support aids to safety warnings. In electric vehicles, these interfaces further help the driver to understand the status of the vehicle with regard to its charging requirements, range, and battery lifetime condition. Requirements for these automotive HMI systems are in general broad and manifold, as the target group of drivers is strongly heterogeneous regarding their purpose of driving, mileage, age, gender and education. Targeting taxi drivers in Singapore, however, gives the opportunity to focus on specific requirements, as the purpose of use can be defined in detail. Addressing the geographical boundaries of Singapore, the properties of an electric vehicle such as the state of charge, the passengers and the target group of taxi drivers, allows to develop a purpose-built, integrated automotive HMI.

Settled in an overall engineering task of building an electric taxi for Singapore, we carried out a requirement engineering process and subsequently developed the HMI for the purpose-built electric taxi. We carried out seven requirement studies to address driver and passenger requirements alike. Within these studies, we evaluated the market situation and targeted usability and acceptance issues to derive content and design implications for the automotive HMI system.

We further specified the hardware based on the requirement studies to set up an automotive communication structure. This structure enables the communication between a variety of devices (i.e. the instrument cluster, the central information screen and smart devic- es such as smartphones. The software manages the communication flow in the vehicle between all devices. We thus describe the status of the software development, including all electric vehicle and taxi-related functionalities in the domains of information control and service structure.

During the development process we addressed issues related to engineering and human-computer interaction research. According to common practice in $\mathrm{HCI}$, we started with iterative development cycles early in the concept phase of the vehicle. We further used robust engineering principles [1], to develop an HMI concept that is reliable and works efficiently on an existing automotive platform. In the following, we first present the requirement studies that were carried out. Subsequently, we describe the concept development process, followed by the description of three interface prototypes.

\section{Requirement Studies}

To gather information about the passenger, the taxi driver and the contextual situation, we carried out seven studies (five qualitative and two quantitative studies).

(S1) Experience and understand taxi driving in Singapore: We first interviewed taxi drivers during a halfyear period regarding their needs during a taxi shift and their experiences with passengers. The interviews were analysed and revealed unique attributes of the Singaporean taxi market that affect the way of taxi usage as well as the experience passengers have when using a taxi. Often, a single taxi is used by two drivers, who drive a company-owned taxi in approximately 12hour shifts, resulting in around-the-clock usage. Booking a taxi requires making a call, using a smartphone 


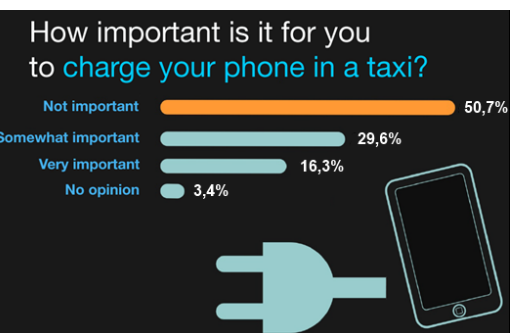

Figure 2: Evaluation of desired features of a Singaporean taxi

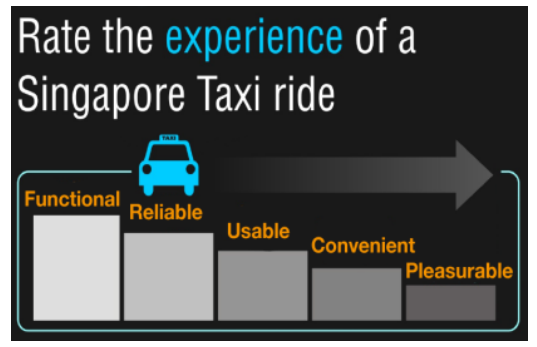

Figure 3: Experience ratings of a taxi ride in Singapore application or sending a text message with your postal code (postal codes in Singapore identify a block of houses rather than parts of a town). The booking request is handled by a dispatch system, which communicates with aftermarket interface solutions that are installed in the taxis (see Figure 1). Hailing a taxi on the street might be difficult, but taxi pick-up points are available all over the city.

The fees for a taxi ride can vary according to peak hour extra payments or area-based fees like for the airport), which are controlled by an electronic road pricing system (ERP). Singapore as a city-state is limited in means of its geographical outline, which induces unique traffic characteristics like peak hour traffic. Regarding the cultural background, Singapore is divided into three major ethnicities: Chinese, Malays and Indians, whereas the group with Chinese background forms the majority [8]. This heterogeneous citizenship and the cultural diversity need to be considered during the whole development process.

(S2) Assessing how taxis should be changed to meet passenger requirements: We conducted twenty qualitative interviews with passengers to gather knowledge about their informational needs and how they use their mobile phones in a taxi. (Figure 2 shows for example that charging a phone is less important, whereas Internet connectivity was highly requested).

(S3) Gathering user experience reports of common taxi situations: We carried out an online survey with 100 taxi users. The passengers rated past taxi rides and express their subjective opinion about taxi driving in Singapore (see Figure 3, which shows that a taxi ride is rated as reliable but not as a pleasurable experience).
(S4) What do passengers do while taxi riding? We conducted an additional survey to focus on what passengers do during a taxi ride, was conducted resulting in smartphone usage being the most frequently mentioned activity.

(S5) Documenting and analysing the behaviour of passengers: We filmed five taxi pick-up points for 3 hours each. The analysis revealed that passengers had particular difficulties in matching a booking confirmation to a certain vehicle.

(S6) Assessing the mobility behaviour of taxis: We carried out a fleet test in Singapore. We handed out smartphones to five taxi drivers to track their overall driving behaviour and the amount of passengers transported. For this, we cooperated with a taxi company and the Land Transport Authority. Taxis in Singapore are a valid supplement to the mass rapid transit or bus system due to reasonable prices. At peak times, the demand for taxis is nevertheless extremely high, especially as we discovered that $75 \%$ of all trips served for the transport of only one person.

(S7) Analysing transportation usage and car sharing: We carried out an online study with 500 participants. The results showed that having a transparent and easy to use taxi sharing system with a transparent fare model is most wanted by the participants. Another aspect mentioned was that passengers would select their sharing partner by gender and ethnicity.

The major requirements out of these studies where: internet connectivity (barely available during a taxi ride), handling taxi booking in an easy way (in the vehicle and outside), smartphone-based control of vehicle 


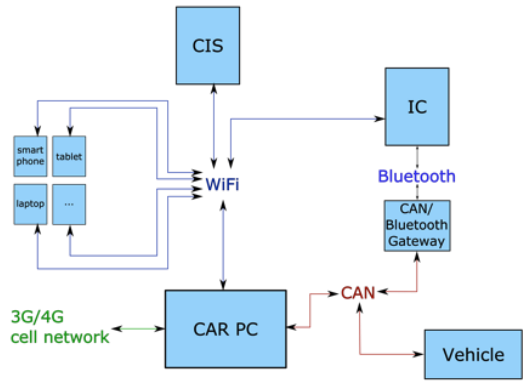

Figure 4: The developed HMI network structure within the vehicle

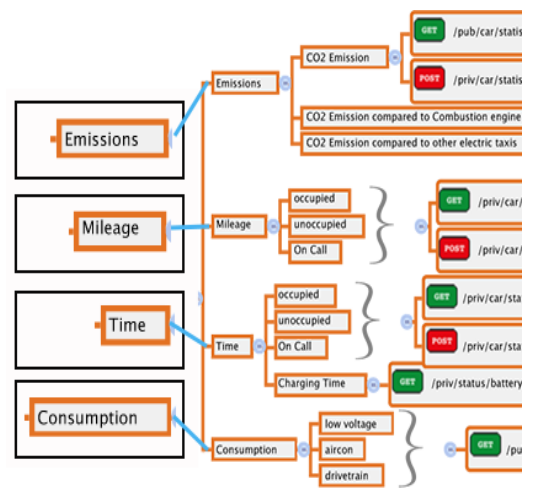

Figure 5: Excerpt of the hierarchical requirement structure for the CIS functionality like climate control for the passenger or locking/unlocking the vehicle for the driver, saving money by sharing taxis, and a suitable interface to support the driver in planning taxi rides like support through range prediction or guidance in optimizing trips in accordance to charging stations.

In order to develop and appropriate HMI system, we first outlined its specifications to build up a flexible and expandable communication system for the vehicle. According to the major requirements, this system should contain multiple devices and interfaces for the communication with the drivers and passengers, as well as a new approach to dispatch systems that allows for taxi sharing. Due to the mobile devices included in the automotive communication system, we defined security as a further major requirement. Thus, we will go into detail about the concept development and the integrated devices in the next section.

\section{Concept Development}

The definition of the hardware is based on our previous experience in developing automotive HMI, on the requirements from the conducted studies (connectivity and informational demand), and on technical boundaries that arose in discussion with the engineers who are responsible for the vehicle structure. For the hardware, our goals were to ensure a feature-rich and fully connected HMI system. We decided for a centralized Car PC with a multitude of I/O possibilities that works as backbone for all peripheral smart devices (see Figure 4 for the system overview and connection details). The Controller Area Network (CAN) is widely used in the automotive context [5] and serves as a wired serial communication framework. The CAN bus connects the critical components associated with driving such as the motor control with the other functions of the vehicle like battery management. To access the driving and control-related data, the HMI system of the vehicle has two gateways to the CAN bus. One is a CAN-Bluetooth gateway that connects the instrument cluster directly to the CAN bus to allow immediate and reliable transfer of safety critical real-time information (for further details see [6]). The other gateway is the Car PC that makes the data on the CAN bus available to the smart devices through a service-oriented architecture. To support flexible and secure connectivity between devices, we implemented two physically separated Wi-Fi networks. This gives access to information on the Car PC and access to the devices. This is advantageous as depending on the source of the device, either the driver or the passengers can use different web-services [3]. The devices are divided into three different groups:

Central information screen (CIS) - Due to ergonomic issues (an input device for the driver needs to be in reach) and that the taxi passengers also need to be informed about the trip, we decided to include a touchsensitive CIS located in the central console. It serves as an input device for controlling all internal functions of the car and informs about the fees and trip details. Figure 5 documents in an excerpt the complexity of the correlating requirements for the CIS.

Instrument cluster (IC) - The IC is dedicated to display all safety-relevant information to the driver, provide additional electric vehicle-related information like an adaptive range prediction and support the driver with taxi-related functionality such as booking details. Both, the IC and CIS can show and process information. For this, we decided to use Android-based systems that communicate with the Car PC bidirectional. 


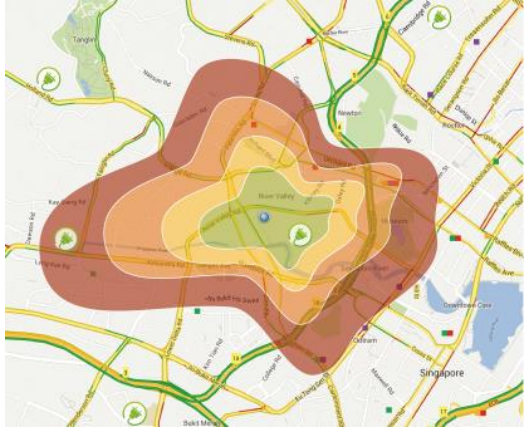

Figure 6: Range prediction concept with geographical references

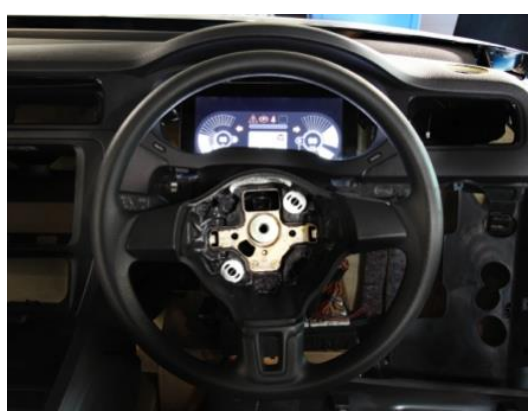

Figure 7: Seatbox IC Prototype Setup to evaluate the UI design

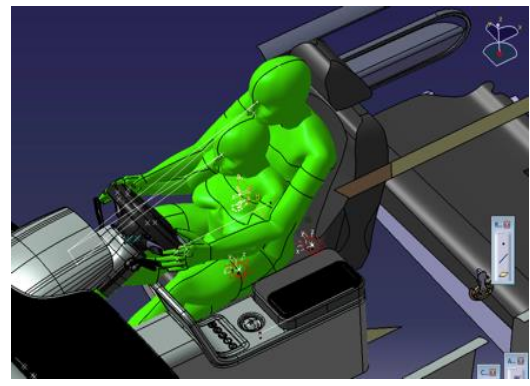

Figure 8: CAD-based ergonomic analysis
Smart devices - Devices such as tablets and smartphones provide vehicle-related information and service control to the driver and the passengers. The devices connect via the Wi-Fi network (see Figure 4) and for example give access the control of the entertainmentor booking system via web services.

\section{HMI Prototypes}

One of the requirements we assessed in our studies was the mobile connectivity demand of passengers and drivers. As the telecommunication infrastructure in Singapore is well developed and used intensively, we decided to implement a mobile Internet connection for all live services, such as information about the estimated arrival time of the taxi to be transmitted to the waiting customers. The cellular connection is shared with al connected devices via the internal Wi-Fi of the vehicle with high priority to the CIS and IC and a lower priority to the passengers' smart device. This enables the devices to access information not provided by the Car PC and to be updated on the fly without the need of local maintenance, as the standing time of a Singaporean taxi is limited due to almost 24 hours of daily use.

Prototype for the CIS - One of the main purposes of the CIS was the requirement to support the driver and passengers' informational needs. We decided, due to visibility and control requirements, to combine both information sources in one interface. For the driver we implemented a dispatch system that allows for accepting and declining booking requests. When the driver accepts a new booking, the pick-up point is automatically entered into the navigation system as the next destination. The interface also shows nearby charging stations, traffic density and predicted range (Figure 6).
For the passengers, the CIS displays details and provides access to climate- and music control.

Prototype for the IC - The IC is based on Android and is directly connected to the CAN bus via a Bluetooth and accesses all driving-related information. Besides speed, torque and odometer, the interface objects that are related to the electric vehicle characteristics (range, power, charging process and energy meter) are displayed. This encloses an appropriate data communication with the vehicle network, as well as a proper interface design according to common practice [4],[9]. Figure 7 shows the evaluation of the IC in a seating box while Figure 8 shows the computer-aided design evaluation of the visibility of the interfaces.

Prototype for a smart device - The driver's smart device can also be used to control functions when in range of the car like the driving light. The smart device app further provides a keyless entry functionality using a Bluetooth Class 2 connection with a max range of 10 meters (Figure 9). This makes it especially easy to transfer the rights to open and use the vehicle between different drivers and also give passengers access to open the otherwise locked rear luggage compartment. For the passenger we developed a taxi sharing application to address the requirement of cheaper and faster taxi bookings. The app is designed to quickly find fellow passengers to a certain destination (Figure 10).

First findings from evaluating the prototypes were that we determined a reduced usability of the CIS. We needed to adjust the font size and defined the upper screen half as a fixed interaction area for the driver to display information for the driver and the passenger similarly. Regarding the IC we were faced with ergo- 


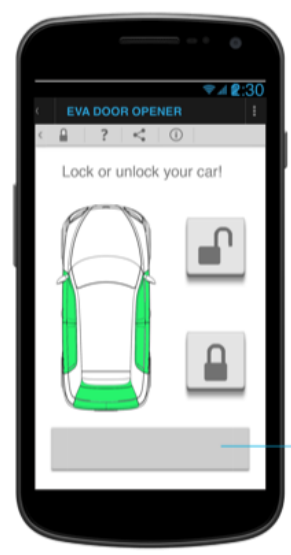

nomic issues as the visible area was small and the changing placement of the seat continuously influenced the visibility of the IC. We replaced the IC once to increase the resolution and the visible array. Taking a look back, the most challenging endeavour was to transfer results from the requirement phase to the system development. It needs to be considered that engineering requirements as well as a fast pace development process reduced the functionalities of the HMI system.

\section{Conclusion}

The main goal of this project is to build a fully functional prototype of an automotive HMI system based on detailed requirements. We have carried out in-depth requirement studies and developed a proper communi-

\section{References}

[1] Amditis, A., Kubmann, H., Polychronopoulos, A., Engstrom, J., and Andreone, L. System architecture for integrated adaptive HMI solutions. In Intelligent Vehicles Symposium, IEEE (2006), 388-393.

[2] Bach, K.M., Jæger, M.G., Skov, M.B., and Thomassen, N. G. You can touch, but you can't look: interacting with in-vehicle systems. In Proc. of $\mathrm{CHI}^{\prime} 08, \mathrm{ACM}$ (2008), 1139-1148.

[3] Broy, M. Challenges in automotive software engineering. In Proc. of Software Engineering, ACM (2006), 33-42.

[4] Green, P. In-vehicle UI standards. In Adjunct Proc. of AutomotiveUI'12, (2012).

[5] International Organization for Standardization cation infrastructure to enable a broad variety of services. We further developed and tested smart device applications and interface prototypes. The evaluation of the prototypes showed ergonomic limitations (visibility, reachability), whereas we see the need for further evaluations when integrating all concepts in the targeted EV to address safety- and usability-related issues while driving in particular.

\section{Acknowledgements}

This work was financially supported by the Singapore National Research Foundation under its Campus for Research Excellence and Technological Enterprise (CRE ATE) programme. We would further like to thank Rahul Gujarathi, Goran Marinkovic, Inka Goethe and Andreas Schwab for their support.

(ISO). ISO 11898-4:2004 Road vehicles - Controller area network (CAN) (2004).

[6] Osswald, S., Sheth, P., Tscheligi, M. Hardware-inthe-Loop-Based Evaluation Platform for Automotive Instrument Cluster Development (EPIC), In Proc. of EICS'13, ACM (2013), in press.

[7] Schmidt, A., Dey, A. K., Kun, A. L., and Spiessl, W. Automotive user interfaces: human computer interaction in the car. In CHI EA'10, ACM (2010), 3177-3180. [8] Singapore Department of Statistics. Population Trends 2012. (2012) http://www.singstat. gov.sg/ [9] Strömberg, H., Ericsson, J., Andersson, P., Karlsson, M., Almgren, S., and Nabo, A. Driver interfaces for electric vehicles. In Proc. of AutomotiveUI'11, ACM (2011), 177-184

Figure 10: Taxi sharing application 